\section{Prevalência de fatores de risco da síndrome metabólica em adultos com síndrome de Down na cidade de Pelotas}

\author{
Prevalence risk factors metabolic syndrome \\ in adults with Down syndrome from the \\ city of Pelotas
}

Angélica Xavier Kalinoski ${ }^{1}$

Alexandre Carriconde Marques ${ }^{2,3}$

Laura Garcia Jung ${ }^{1}$

Gabriela Brisolara Xavier ${ }^{1}$

\section{Resumo}

Compreender fenômenos relacionados com a Síndrome de Down (SD) auxilia a implementação de programas de promoção à saúde para esse público, melhorando a qualidade de vida. O objetivo desse trabalho foi descrever a prevalência de SM de adultos com SD maiores de 18 anos. A população desse estudo foi constituída por 30 adultos com SD selecionados intencionalmente, com média de idade de 25,5 ( $\mathrm{DP}=5,6)$ e prevalência de SM de 23,3\% ( $\mathrm{n}=7$ ). Foi utilizado o programa SPSS 13.0 for Windows, para as análises estatísticas sendo adotado um nível de significância de $5 \%(\mathrm{p} \leq 0,05)$. O estudo baseou-se nas análises do NCEP-ATP III (2002), que observou os seguintes fatores de risco para a SM: triglicerídeos, HDL, glicemia de jejum, circunferência abdominal e pressão arterial. Na população masculina, os fatores de risco com maiores prevalências foram os triglicerídeos e colesterol HDL com 42,1\% ( $n=8)$ e circunferência abdominal com $47,4 \%(n=9)$. $\mathrm{Na}$ população feminina, os fatores de risco de maiores prevalências foram o colesterol HDL com $63,3 \%(n=7)$. Assim percebe-se a necessidade de um aprofundamento maior nos estudos relacionados com AF e essa população, auxiliando na redução dos riscos para SM.

\section{Palavras-chave}

Adultos; Síndrome de Down; Síndrome metabólica; Atividade Física; Estilo de vida.

\begin{abstract}
Understand phenomena related to Down Syndrome (DS) assists the implementation of health promotion programs to this population, improving their quality of life. The aim of this work was to describe the MS prevalence in adults with DS over 18 years. The study population consisted of 30 adults with $D S$, intentionally selected, with average age 25,5 (DP=5,6) and $M S$ prevalence of 23,3\% (n=7). The software SPSS 13.0 for Windows was used for statistical analyses, being adopted a level of significance of $5 \%(p \leq 0,05)$. The study was based on NCEP-ATP III (2002) that observed the following risk factors to MS: triglycerides, HDL, fasting glucose, abdominal circumference and blood pressure. In male population, risk factors with higher prevalence were triglycerides and HDL cholesterol with $42,1 \%(n=8)$ and abdominal circumference with 47,4\% (n=9). In the female population, risk factor with highest prevalence was cholesterol HDL with 63,3\% ( $n=7)$. Therefore, it is noted a need of further studies related to $P A$ and this population, helping to reduce risks for $M S$.
\end{abstract}

\section{Keywords}

Adults; Down syndrome; Metabolic syndrome; Physical activity; Lifestyle
Rev Bras Ativ Fis Saúde p. 396-402 DOI: http://dx.doi.org/10.12820/23171634.2012v17n5p396

1 Escola Superior de Educação Física, Universidade Federal de Pelotas, Pelotas, $R S$, Brasil

2 Escola Superior de Educação Física, Universidade Federal de Pelotas, Pelotas, RS, Brasil

3 GEEAF - Grupo de Estudos em Epidemiologia da Atividade Física 


\section{INTRODUÇÃO}

A Síndrome de Down (SD) é um acidente genético que acontece na divisão cromossômica das células ${ }^{1}$. Pessoas com SD possuem algumas características físicas típicas da síndrome que podem ser consideradas desvantajosas para a saúde, entre elas um crescimento físico mais lento, $24 \%$ menor do que as outras crianças. Devido esse crescimento, podem começar a engordar, consequentemente ocasionando excesso de peso $^{2}$.

Em função dessas características e outras variáveis relacionadas ao estilo de vida dessa população em especial o baixo nível de atividade física, pessoas com SD estão suscetíveis a desenvolver uma série de doenças crônicas não transmissíveis $(\mathrm{DCNT})^{2}$. Essas doenças compreendem majoritariamente doenças cardiovasculares, diabetes, câncer e doenças respiratórias crônicas ${ }^{3}$.

Algumas dessas doenças, como diabetes mellitus tipo II e doenças cardiovasculares, estão relacionadas com alterações no metabolismo ${ }^{4}$. As pessoas que as possuem apresentam fatores de risco para a Síndrome Metabólica (SM), a qual foi descrita em 1988 por Gerald Reaven, como a intolerância a glicose, resistência a insulina ou diabetes mellitus, incluindo dois fatores ou mais como a hipertensão, triglicéride plasmático elevado, HDL - colesterol baixo e obesidade central ${ }^{5}$.

Os fatores de risco para a SM tem relação com atividade física, pois estudos epidemiológicos mostram forte relação entre inatividade física e presença de fatores de risco cardiovascular como hipertensão arterial, diabetes, resistência à insulina, dislipidemia e obesidade. ${ }^{6}$

No entanto, há uma lacuna de estudos que explorem a relação entre SM em pessoas com SD levando em consideração o estilo de vida dessa população. $\mathrm{O}$ presente estudo teve como objetivo descrever a prevalência de $\mathrm{SM}$ e as características de estilo de vida de adultos com SD maiores de 18 anos.

\section{MATERIAIS E MÉTODOS}

O presente trabalho é caracterizado como um estudo transversal ${ }^{7}$. Para constituir essa amostra foram selecionadas, intencionalmente, todas as pessoas com SD, maiores de 18 anos, cujos pais ou responsáveis assinaram o termo de consentimento livre e esclarecido. Sabe-se que uma das limitações do estudo é a falta de dados relacionados a essa população, não há um levantamento de quantas pessoas com SD moram na cidade de Pelotas. Muitas dessas pessoas não estão em instituições ou projetos, dificultando o acesso para entrar em contato. Nesse estudo não houve critérios de exclusão, e os critérios de inclusão era ter SD e ser maior de 18 anos e residir em Pelotas.

Para o recrutamento foi feito um primeiro contato com as instituições (escolas) e projetos de extensão da Universidade Federal de Pelotas (UFPel) que atendem adultos com SD, após, foi solicitada a presença dos responsáveis para que assinassem o termo de consentimento livre e esclarecido (TCLE) para a coleta de dados. O projeto foi submetido ao Comitê de Ética em Pesquisa da Escola Superior de Educação Física (ESEF) da Universidade Federal de Pelotas (UFPel) e aprovado com protocolo no $138 / 2010$.

As variáveis antropométricas foram coletadas com os indivíduos descalços e com o mínimo de roupa possível. A estatura foi mensurada por meio de um estadiômetro; a massa corporal foi obtida em uma balança digital Filizola. Para a cir- 
cunferência abdominal, foi utilizada uma fita métrica flexível não elástica, estando o indivíduo com a região, a ser avaliada, descoberta. Posicionou-se a fita no meio da distância entre a crista ilíaca e a face externa da última costela - por ser o índice antropométrico mais representativo da gordura intra-abdominal e de aferição mais simples e reprodutível ${ }^{8}$.

A aferição da pressão arterial foi obtida por método de ausculta com a utilização de estetoscópio e esfigmomanômetro. A aferição foi feita após 5 minutos de repouso, na posição sentada, braço direito apoiado sobre uma mesa, certificando-se que o avaliado não estivesse com a bexiga cheia, não tivesse praticado exercícios físicos e nem ingerido bebidas alcoólicas, café, alimentos ou fumado até 30 minutos antes da medida. $\mathrm{O}$ manguito foi colocado firmemente, centralizando a bolsa de borracha sobre a artéria braquial.

Para determinação da pressão arterial sistólica (PAS), foi considerada a fase I de Korotkoff e a fase $\mathrm{V}$ para a pressão arterial diastólica (PAD), utilizando-se como critério para determinação da pressão sistólica o momento do aparecimento do primeiro som (Fase I de Korotkoff) que se intensifica com o aumento da velocidade de desinsuflação e, para a determinação da pressão diastólica, o desaparecimento do som (Fase $\mathrm{V}$ de Korotkoff) ao persistirem os batimentos até o nível 0, determinar-se a pressão diastólica no abafamento dos sons (Fase IV de Korotkoff) 9 .

Os exames para a obtenção de amostra sanguínea foram realizados no Laboratório do Hospital Escola da Universidade Federal de Pelotas, após 12 horas de jejum e repouso de 30 minutos antes da coleta no período da manhã. As análises bioquímicas foram efetuadas pelo laboratório do Hospital Escola. Foram utilizadas as definições estabelecidas pela National Cholesterol Education Program's Adult Treatment Panel III (NCEP-ATP III). Os valores de referencia adotados foram: glicemia de jejum ( $\geq 110 \mathrm{mg} / \mathrm{dL}$ ), triglicerídeos ( $\mathrm{TG} \geq 150 \mathrm{mg} / \mathrm{dL})$, colesterol HDL ( $<0 \mathrm{mg} / \mathrm{dl}$ para homens e $<50 \mathrm{mg} / \mathrm{dl}$ para mulheres).

Para avaliação do estilo de vida e da atividade física da população avaliada nesse trabalho, foi aplicado um questionário, o mesmo foi adaptado a partir do roteiro da entrevista semiestruturada utilizada no estudo de Marques \& Nahas ${ }^{10}$. O instrumento foi dividido em nove dimensões: dados de identificação; dados familiares; dados socioeconômicos; informações educacionais; relações de traba1ho; atividade Física habitual e preferência no lazer; atividade física; prática de esportes; percepção de saúde. Para esse estudo o questionário foi aplicado com os pais ou responsáveis.

As variáveis antropométricas, pressão arterial, bioquímica sanguínea foram obtidas de forma intervalar, para as análises foram de forma dicotômicas. Para a análise dos dados, foram utilizados recursos de estatística, médias, distribuição de freqüências (absoluta e relativa), desvio padrão. Com relação às análises estatísticas, foi utilizado o programa SPSS 13.0 for Windows, sendo adotado um nível de significância de $5 \%(\mathrm{p} \leq 0,05)$.

\section{RESULTADOS}

A amostra foi constituída de 30 adultos com SD, com uma média de idade de 25,5 anos (DP=5,6; 19 - 39), sendo 19 homens 63,3\% (n=19) com média de idade de 25,9 anos ( $\mathrm{DP}=5,6 ; 19$ - 39) e 11 mulheres 36,7\% ( $\mathrm{n}=11)$ com média de idade de $24,8$ anos ( $\mathrm{DP}=5,7 ; 20$ - 39). A prevalência de $\mathrm{SM}$ foi de $23,3 \%$ ( $\mathrm{n}=7)$, a tabela 1 
caracteriza a amostra estratificada por sexo de acordo com os fatores de risco para Síndrome Metabólica:

Tabela 1 - Caracterização da amostra de acordo com os fatores de risco para Síndrome Metabólica.

\begin{tabular}{lcc}
\hline Prevalência dos fatores de risco para Síndrome Metabólica & $\mathrm{N}$ & $\%$ \\
\hline Homens $(\mathrm{n}=19)$ & & \\
\hline Com $\mathrm{SM}^{*}$ & 05 & 26,3 \\
\hline Triglicerídeos $\geq 150 \mathrm{mg} / \mathrm{dl}$ & 08 & 42,1 \\
\hline $\mathrm{HDL}<40 \mathrm{mg} / \mathrm{dl}$ & 01 & 42,1 \\
\hline Glicemia de jejum $\geq 110 \mathrm{mg} / \mathrm{dl}$ & 09 & 5,3 \\
\hline Circunferência Abdominal $>102 \mathrm{~cm}$ & 05 & 47,4 \\
\hline PA S $\geq 130 \mathrm{mmHg}$ & 02 & 26,3 \\
\hline PA D $\geq 85 \mathrm{mmHg}$ & & 10,5 \\
\hline Mulheres $(n=11)^{*}$ & 02 & 18,2 \\
\hline Com SM* & 01 & 9,1 \\
\hline Triglicerídeos $\geq 150 \mathrm{mg} / \mathrm{dl}$ & 07 & 63,3 \\
\hline HDL $<50 \mathrm{mg} / \mathrm{dl}$ & 05 & 45,5 \\
\hline Circunferência Abdominal> $88 \mathrm{~cm}$ & 01 & 9,1 \\
\hline PA S $\geq 130 \mathrm{mmHg}$ & 02 & 18,2 \\
\hline PA D $\geq 85 \mathrm{mmHg}$ & 07 & 23,3 \\
\hline PREVALÊNCIA DE SM $(n=30)$ & & \\
\hline
\end{tabular}

*SM = Síndrome Metabólica

* *Nenhuma das mulheres apresentou glicemia de jejum alterada.

Em relação ao nível socioeconômico (ABEP, 2010) ${ }^{11}$ : entre os homens, 5,3\% $(n=1)$ representam a classe $A ; 10,5 \%(n=2)$ representam a classe $B ; 31,6 \%(n=6)$ representam a classe $C ; 52,6 \%(n=10)$ representam a classe $D$. Entre as mulheres, $27,3 \%(n=3)$ representam a classe $B ; 36,4 \%(n=4)$ representam a classe $C ; 36,4 \%$ $(\mathrm{n}=4)$ representam a classe D. O nível sócio econômico demonstrou uma prevalência de $\mathrm{SM}$ maior nas classes $\mathrm{C} ; 6,6 \%(\mathrm{n}=2)$ e $\mathrm{D} ; 9,9 \%(\mathrm{n}=3)$, em relação às classes A e B que ambas foram representadas por 3,3\% (n=1).

De acordo com os dados escolares, viu-se que 78,9\% ( $n=15)$ dos homens frequentam escola; desses, $80,0 \%(\mathrm{n}=12)$ estudam em escolas especiais ou projetos de alfabetização e 20,0\% (n=3) estudam em escolas regulares. Constatou-se que, entre os homens, apenas $10,5 \%(n=2)$ sabem ler e escrever. Já em relação às mulheres, viu-se que $81,8 \%(n=9)$ frequentam escola; dessas, todas estudam em escolas especiais ou projetos de alfabetização. Entre elas, $72,7 \%(\mathrm{n}=8)$ sabem ler e escrever.

Tratando-se da atividade de trabalho, 94,7\% ( $\mathrm{n}=18)$ dos homens nunca trabalharam e 5,3\% ( $n=1)$ trabalham, sendo que o tipo de trabalho relatado foi de serviços gerais de laboratório. E nenhuma das mulheres relatou experiência de trabalho.

Com relação ao comportamento sedentário, viu-se que a média de tempo que os homens assistem à televisão foi de $216 \mathrm{~min} / \mathrm{dia}(\mathrm{DP}=2,02)$ e, nos finais de semana, foi de $270 \mathrm{~min} / \mathrm{dia}(\mathrm{DP}=2,77)$. Entre as mulheres, a média foi de $246 \mathrm{~min} /$ dia $(\mathrm{DP}=3,02)$ e nos finais de semana foi de $276 \mathrm{~min} / \mathrm{dia}(\mathrm{DP}=3,32)$. Também pode constatar que a média de tempo que os homens utilizavam o computador foi de $90 \mathrm{~min} / \mathrm{dia}(\mathrm{DP}=1,16)$ e, durante os finais de semana, foi de $180 \mathrm{~min} / \mathrm{dia}$ $(\mathrm{DP}=1,6)$; entre as mulheres, a média foi de $90 \mathrm{~min} / \mathrm{dia}(\mathrm{DP}=1)$ e nos finais de semana foi de $105 \mathrm{~min} / \mathrm{dia}(\mathrm{DP}=0,9)$. 
Tabela 2 - Atividade habitual e atividade mais realizada no lazer.

\begin{tabular}{lcccc}
\hline ATIVIDADE HABITUAL & $\begin{array}{c}\text { Homens } \\
\mathrm{N}(19)\end{array}$ & $\%$ & $\mathrm{~N}(11)$ & $\%$ \\
\hline Assistir à TV & 5 & 26,3 & 4 & 36,4 \\
\hline Escutar música & 6 & 31,6 & - & - \\
\hline Passear com a família ou amigos & 2 & 10,5 & 3 & 27,3 \\
\hline Ler ou desenhar & 1 & 5,1 & 1 & 9,1 \\
\hline Conviver e conversar com amigos & 1 & 5,1 & 2 & 18,2 \\
\hline Ajudar nas tarefas domésticas & 2 & 10,5 & 1 & 9,1 \\
\hline
\end{tabular}

Quando relataram o número de dias que faziam caminhadas rapidamente, a média entre os homens foi de três dias $(\mathrm{DP}=1,9)$ e a média de horas dessas caminhadas foi de 20 min $(\mathrm{DP}=0,26)$. Entre as mulheres, a média foi de 3,78 dias $(\mathrm{DP}=1,56)$ de caminhada com uma média de duração de 33 min $(\mathrm{DP}=0,38)$.

De acordo com o observado sobre a prática de esporte entre os homens, nove praticam natação, sete fazem dança, cinco jogam futebol e um pratica judô. Constatou-se que a média de dias em que praticam esses esportes foi de 2,9 dias $(\mathrm{DP}=1,3)$ com uma média de duração de $60 \mathrm{~min}(\mathrm{DP}=0,39)$. Entre as mulheres, cinco praticam natação, oito fazem dança e uma joga futebol, perfazendo a média de 2,6 dias $(\mathrm{DP}=0,51)$ de prática dessas atividades, com média de duração de 60 $\min (\mathrm{DP}=0,00)$.

Tabela 3 - Pratica de atividade física e de esporte.

\begin{tabular}{lccccc}
\multicolumn{1}{c}{ ATIVIDADE FísICA } & Homens & \multicolumn{3}{c}{ Mulheres } \\
& $\mathrm{N}(19)$ & $\%$ & $\mathrm{~N}(11)$ & $\%$ \\
\hline Pratica alguma atividade física & 16 & 81,6 & 10 & 90,9 \\
\hline Pratica algum esporte & 15 & 76,5 & 8 & 72,8 \\
\hline
\end{tabular}

\section{DISCUSSÃO}

Entre os fatores de risco da SM estudados, era esperado que a circunferência abdominal fosse o principal fator de risco, o que foi confirmado no sexo masculino, dos quais $47,4 \%(n=9)$ estavam acima do indicado. Porém, entre o sexo feminino o principal fator de risco encontrado foi o HDL, onde $63,3 \%(n=7)$ estavam abaixo do adequado, já considerando que a indicação do valor mínimo de HDL é maior no sexo feminino.

Analisando os dados sem o fator de risco circunferência abdominal, a prevalência de SM em adultos com SD foi de $10,0 \%(n=3)$. A explicação para tal fato deve-se ao crescimento ósseo dessa população, o qual diminui enquanto a massa corporal continua a aumentar. Dessa forma, é difícil determinar padrões para classificação da circunferência abdominal nessa população ${ }^{1}$.

Na população estudada, a prevalência de SM foi de 23,3\%. São escassos os estudos com esse tema nessa população específica, porém em outras populações a prevalência vem sendo descrita, como por exemplo, em adultos norte americanos, que é de $23 \%$, já em pessoas com psoríase é $28 \%$, em pessoas com diabetes tipo 2 essa prevalência é de $62 \%{ }^{12-13}$.

Em relação aos triglicerídeos e a hipertensão arterial, há alguns estudos que mostram que pessoas com SD têm risco aumentado para esses fatores ${ }^{14,15}$. Em um estudo que avaliou 52 adultos com SD com o objetivo de determinar se essa popu- 
lação possui menos aterosclerose do que adultos sem $\mathrm{SD}$, no qual foram avaliados alguns fatores de risco para a aterosclerose como os triglicerídeo com uma média de $126,5 \mathrm{mg} / \mathrm{dl}(\mathrm{DP}=52,2)$, a pressão arterial sistólica $116 \mathrm{mmHg}(\mathrm{DP}=15)$ e a diastólica $59 \mathrm{mmHg}(\mathrm{DP}=10)^{16}$.

O nível de atividade física na população estudada apresentou valores satisfatórios como mostram os resultados. Esse alto índice é devido ao fato da população estudada estar participando de projetos de extensão e estarem em escolas. Pois, quando uma pessoa com SD está na escola ou em projetos, as oportunidades são aumentadas, inclusive para a prática de atividade física. Em relação aos dados escolares dos homens, 78,9\% $(\mathrm{n}=15)$ frequentam escola. Já em relação às mulheres, viu-se que $81,8 \%(n=9)$ frequentam escola.

Ao analisar dias e tempo de caminhada, como mostraram os resultados, as médias encontradas também foram satisfatórias, pois, por estarem inseridos em projetos de atividade física e matriculados em escolas, necessariamente eles têm que realizar algum deslocamento. Por outro lado, os familiares dos participantes de projetos de atividade física, por estarem presentes durante as atividades desenvolvidas, acabam entendendo a importância da atividade física e aderindo a uma rotina mais ativa, beneficiando tanto ao filho quanto os demais membros da família.

A prática esportiva foi outro índice satisfatório, pois se constatou que a maioria realiza alguma prática esportiva. Como já foi exposto anteriormente, isso se deve ao fato dessas pessoas terem a oportunidade de participar de projetos que visam à atividade física, contemplando os esportes.É interessante ressaltar ainda que, após a coleta de dados do estudo, algumas pessoas com SD começaram a participar de atividades físicas.

Uma limitação para o recrutamento da amostra foi a falta de contato com o grupo estudado, cujo primeiro contato foi feito através de instituições de ensino. Justifica-se assim, a alta prevalência de participantes inseridos na escola da amostra estuda. Sabe-se que são poucos os adultos com SD que frequentam escola e poucas instituições atendem essa população, o que dificulta o contato com essa população que passa a maior parte do tempo em suas casas.

\section{CONCLUSÃO}

Neste estudo, foi verificado que grande parte da amostra pratica algum tipo de atividade física, podendo melhorar a qualidade de vida e auxiliar na manutenção dos fatores de risco para a SM. Tratando-se de SM sempre devemos levar em consideração as variáveis como genética, alimentação, ingestão de medicamentos e outros fatores que são fundamentais para a saúde.

Com bases nos dados e de acordo com a literatura, percebe-se a necessidade de maiores estudos nessa área, pois ainda são escassos. Também é importante fomentar projetos que oportunizem a $\mathrm{AF}$ para pessoas com $\mathrm{SD}$, melhorando, assim, a qualidade de vida desses indivíduos, pois, além da $\mathrm{AF}$ ser um hábito saudável, ela tem um importante papel social na vida dessas pessoas, melhorando a qualidade de vida.

\section{Contribuiç̧ões dos autores}

Elaboração do projeto e instrumento: Angélica Kalinoski, Laura Jung, Alexandre Marques. Coleta de dados: Angélica Kalinoski, Laura Jung, Alexandre Marques. Análise de dados: Angélica Kalinoski e Alexandre Marques, Gabriela Brisolara Xavier. Redação do estudo: Angélica Kalinoski, Laura Jung, Alexandre Marques, Gabriela Brisolara Xavier. 


\section{REFERÊNCIAS}

1. Rogers P,Coleman M. Atencion médica en el Sindrome de down: unplanteamento de medicina preventive. Barcelona: Fundació Catalana Síndrome Down, 1994.

2. Marques AC. O perfil do estilo de vida de pessoas com Síndrome de Down e normas para avaliação da aptidão física. Porto Alegre: Escola de Educação Física, Universidade Federal do Rio Grande do Sul, 2008.

3. Achutti A, Azambuja MIR. Doenças crônicas não-transmissíveis no Brasil: repercussões do modelo de atenção à saúde sobre a seguridade social. Ciência \& Saúde Coletiva 2004;9:833-40.

4. Lottenberg SA, Glezer A, Turatti LA. Metabolic syndrome: identifying the risk factors. J Pediatr 2007;83:204-208.

5. Reaven GM. Role of insulin resistance in human disease. Diabetes. 1988;37: 1595-607.

6. Ciolac, EG, Guimarães, GB. Exercício físico e síndrome metabólica. Rev. Bras. Med. Esporte 2004;10:319-324.

7. Pereira M. Epidemiologia: teoria e prática. Rio de Janeiro: Guanabara Koogan, 1995.

8. I Diretriz Brasileira de Diagnóstico e Tratamento da Síndrome Metabólica. Arquivos Brasileiros de Cardiologia. 2005;84:3-28.

9. Sociedade Brasileira de Cardiologia - SBC. IV Diretrizes Brasileiras de Hipertensão Arterial. Arq Bras Cardiol 2004;82:1-14.

10. Marques A, Nahas M. Qualidade de vida de pessoas portadoras de Síndrome de Down, com mais de 40 anos, no Estado de Santa Catarina. Rev Bras Cien Mov. 2003;11:55-61.

11. http://www.abep.org/novo/index.htm. Acessado em 15 de dezembro de 2011.

12. Agirbasli M, Cakir S, Ozme S, Ciliv G. Metabolic syndrome in Turkish children and adolescents. Metabolism. 2006;55:1002-6.

13. Castillo EH, Borges G, Talavera JO, et al. Body mass index and the prevalence of metabolic syndrome among children and adolescents in two Mexican populations. J Adolesc Health 2007;40:521-6.

14. Martínez R, Ibáñez A, Gómez P, et al. Utilidad del Perímetro Abdominal como Método de Cribaje del Síndrome Metabólico en las Personas con Hipertensión arterial. Rev Espa Salud Pública 2010;84:215-22.

15. Silva D, Santos J, Martins C. Avaliação da Composição Corporal em Adultos com Síndrome de Down. Arquivos de Medicina 2006;20:103-10.

16. Draheim CC, Geijer JR, Dengel DR. Comparison of intima-media thickness of the carotid artery and cardiovascular disease risk factors in adults with versus without the Down syndrome. Am J Cardiol 2010;106:1512-6.

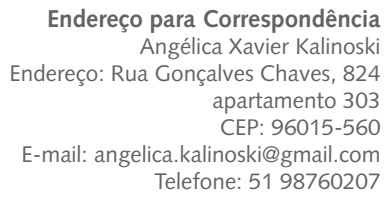

